\title{
Optimization of a heat engine based on a dissipative system
}

\author{
Michael Mozurkewich a) and R. Stephen Berry \\ Department of Chemistry and the James Franck Institute, The University of Chicago, Chicago, Illinois 60637
}

\begin{abstract}
A new class of heat engine is analyzed in which the working fluid operates in a dissipative process, never in equilibrium. The conditions are found for stability and for the generation of work. Then the optimal path is found for operating the general dissipative engine by means of optimal control theory. The optimal cycle consists of arcs of constant power and of approximately instantaneous adiabats. If the heat flow is a function of temperature only, then the constant power arcs become isotherms. An upper bound is found to the power output. Two examples are worked out in detail: a light-driven dissipative engine whose absorption is a step function of temperature, and a lightdriven dissipative engine whose working fluid undergoes a chemical reaction (isomerization), absorbing light in the isomeric form favored at high temperatures.
\end{abstract}

PACS numbers: $05.70 . \mathrm{Ln}, 44.90 .+\mathrm{c}, 89.30 .+\mathrm{f}$

\section{INTRODUCTION}

Both heat engines and dissipative thermodynamic systems such as oscillating chemical reactions make use of a flow across a drop in potential. The heat engine uses a flow of heat across a temperature drop to produce work. In a purely dissipative system the flow maintains instabilities or oscillations and all of the potential work is dissipated. In this paper we consider a heat engine based on a dissipative system and show how it is possible to produce work from the dissipative system's tendency toward oscillations.

A conventional heat engine is periodically brought into contact with its external reservoirs by means of some controlling mechanism. In contrast, the unstable or oscillating system is simultaneously coupled to both external reservoirs in a manner that depends nonlinearly on the state of the system. The heat engine considered here, like the dissipative system, is simultaneously coupled to both reservoirs. Since it cannot be in equilibrium with both reservoirs this engine cannot be operated reversibly.

We make use of the method of finite time thermodynamics to study this engine. In applying this approach we consider only the most important irreversibilities, in this case the heat flows between the engine and its reservoirs. The results provide an upper limit to the efficiency of such an engine. This limit is more realistic than the limit provided by reversible thermodynamics. The results also serve as a guide to more detailed engineering studies.

The dissipative system and the engine based on it, which we consider here is similar to a dissipative system studied by Nitzan and Ross.' They considered a chemical reaction

$$
A \leftrightarrow B,
$$

in which $A$ absorbs light and rapidly relaxes. The heat released flows through the walls of the system into the surroundings. The system is assumed to consist of a single, homogeneous phase which is always in chemical equilibrium and kept at constant volume. Nitzan and Ross found that if

\footnotetext{
'Present address: Hydrocarbon Research Institute, University ParkMC1661, University of Southern California, Los Angeles, California 90089.
}

the formation of $B$ is exothermic it is possible for the system to exhibit multiple steady states when illuminated. Two of these are stable. One of these is at a high temperature with a large amount of $\boldsymbol{A}$. The light is strongly absorbed, thus maintaining the high temperature. The other is at a low temperature with very little $A$ and very little light absorption.

The system which we consider is shown in Fig. 1. It is essentially the same as the system of Nitzan and Ross except for the free piston of mass $m$ and the constant external pressure, $P_{0}$. In Sec. II we show that by allowing the volume to vary we can obtain oscillations between the strongly and weakly absorbing states. The conditions under which this occurs have a simple physical explanation and indicate that work can be extracted from such a system. At this stage of the analysis no means of extracting work, either by working on some other system or through dissipation, is provided. As a result the tendency for the system to behave like an engine manifests itself in more and more rapid motion of the piston.

In Sec. III we consider this system as an engine, i.e., we allow for the extraction of work. This is achieved by requiring the piston to move along a specific path which is maintained by a high-inertia flywheel and determined by a linkage which delivers work to the flywheel reservoir. For example, a crank linkage could be used to convey work from the piston to a rotating shaft which in turn is attached to the flywheel. So long as the energy stored in the flywheel is large compared to the work done in one cycle and either the average rate at which work is removed from the flywheel matches the average power output of the engine or the en-

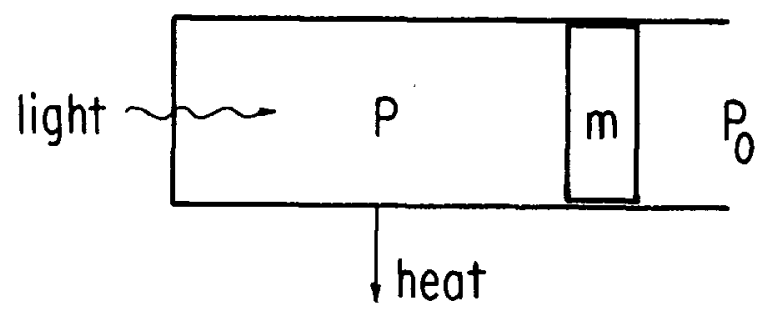

FIG. 1. The dissipative heat engine. The engine is continuously in contact with a light source (high temperature reservoir) and the surroundings (low temperature reservoirl. 
gine-plus-flywheel charge some reservoir of potential energy, e.g., by raising weights, then the shaft rotates at a constant velocity. This is, of course, the usual model for an engine. Under these conditions, the piston then undergoes a periodic motion determined by the nature of the linkage. The linkage and the flywheel determine the piston motion almost completely so long as the flywheel has an inertia much greater than that of the piston, and therefore acts as a work reservoir. For such a system the piston mass is negligible. Engines in which the piston mass is the major inertial component have been examined by Fairén and Ross. ${ }^{2}$ We restrict our consideration here to the more usual system that operates with a flywheel. If the piston motion is governed by the flywheel and linkage, then the effective external pressure on the piston is also determined. The net force on the piston at each instant is the difference between the force developed inside the engine's cylinder and the external force fixed by the linkage. It becomes natural to look upon the linkage as a control variable, allowing one to optimize the time path of the piston.

In Sec. III we determine what piston motion maximizes the power obtained from an engine based on the dissipative system discussed in Sec. II. For this "dissipative" engine the optimal cycle serves a purpose analogous to that of the Carnot Cycle for a reversible engine. It provides a limit for the efficiency of such an engine. Much of the analysis can be carried out in a form so general that it is unnecessary to specify either the working fluid or heat transfer relations. The results provide a relatively easy way of evaluating candidate systems.

In Sec. IV we apply the general results of Sec. III to a specific engine in which the light absorption is a step function of the temperature. An analytic solution is obtained in this case. In Sec. $V$ we replace the discontinuous change in absorption with one produced by a chemical reaction and obtain numerical results for the average power. We then compare the maximum power for this case with that obtained by an engine with a simple, sinusoidal motion.

In Sec. VI we summarize the results and describe how they could be of use in the development of a practical dissipative engine.

\section{STABILITY CONDITIONS FOR THE DISSIPATIVE ENGINE}

The equations of motion for the system shown in Fig. 1 are

$$
\begin{aligned}
& d x / d t=v, \\
& d v / d t=\left[P(x, s)-P_{0}\right] / m, \\
& d s / d t=h(x, s) / T(x, s),
\end{aligned}
$$

where $x$ is the displacement of the piston (proportional to the volume), $s$ is the entropy of the fluid, $P$ and $T$ are the pressure and temperature of the fluid (given by the equations of state), and $h$ is the net heat flow into the system; $h$ depends parametrically on the external temperature. The form of that dependence is not relevant here. The last equation is valid as long as the fluid is homogeneous, is in chemical equilibrium and has high rates of deexcitation for the states produced by the absorption process. In this case we can regard the energy absorbed as appearing as heat at the temperature of the fluid. Then the light absorption process itself can be considered to be outside the boundaries of the system, and the transfer of energy from light to heat is the passage of energy into the system. The irreversibility associated with the heat flow out is also external to the system. Engines of this sort have been termed endoreversible. ${ }^{4}$

Consider the special case in which the heat flow $h$ is zero for all states of the system. Then any displacement of the piston from equilibrium will initiate an adiabatic, reversible process. The phase diagram for the resulting piston motion, shown in Fig. 2(a), is qualitatively the same as that of the harmonic oscillator. The trajectories consist of conservative oscillations; such motion is marginally stable. If the slightest damping term is added to the system, for example a heat flow to the surroundings, the oscillations diminish and the system will return to the steady state as shown in Fig. 2(b). If an appropriate driving term is added we can get unstable oscil-
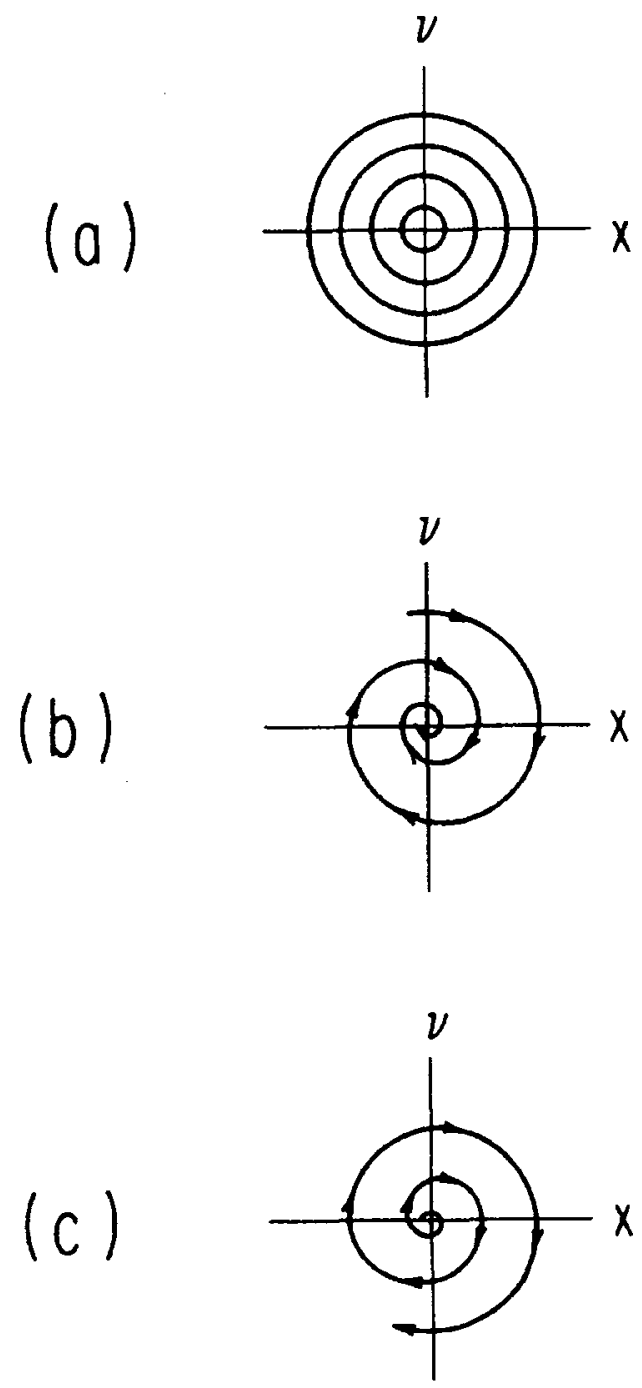

FIG. 2. Possible phase diagrams for the dissipative engine showing projections on the $x, v$ plane of the three types of oscillatory motion: (a) conservative oscillations, (b) damped oscillations, (c) unstable oscillations. 
lations as shown in Fig. 2(c). In this section we determine the conditions that the function $h(x, s)$ must satisfy to produce this unstable behavior.

The system we are considering has a steady state whenever $v=h=0$ and $P=P_{0}$. There may be more than one such point. The stability of a steady state point is determined by the behavior of small perturbations from that state. We can examine the behavior of these perturbations by linearizing the equations of motion about such a point to obtain

$$
\begin{aligned}
& d \Delta x / d t=v, \\
& d v / d t=\left(P_{x} \Delta x+P_{s} \Delta s\right) / m, \\
& d \Delta s / d t=\left(h_{x} \Delta x+h_{s} \Delta s\right) / T .
\end{aligned}
$$

Here $\Delta s$ and $\Delta x$ represent the displacements from the steady state values and $P_{x}$, etc., represent the partial derivatives evaluated at the steady state.

We can now proced to obtain the eigenvalues of this linear system in the usual manner. The eigenvalues $\lambda$ are given by the roots of the characteristic polynomial

$$
0=\lambda^{3}-\left(h_{s} / T \lambda^{2}-\left(P_{x} / m\right) \lambda+\left(P_{x} h_{s}-P_{s} h_{x}\right) / m T .\right.
$$

If the steady state is to be stable all of the coefficients in this equation must be positive. ${ }^{5}$ Since $m$ and $T$ are positive this requires that

$$
\begin{aligned}
& h_{s}<0, \\
& P_{x}<0, \\
& P_{s} h_{x}-P_{x} h_{s}<0 .
\end{aligned}
$$

The Routh-Hurwitz determinant must also be positive. ${ }^{5}$ For this system this condition is

$$
\left|\begin{array}{cc}
-h_{s} / T & 1 \\
\left(P_{x} h_{s}-P_{s} h_{x}\right) / m T & -P_{x} / m
\end{array}\right|>0,
$$

which reduces to

$$
P_{s} h_{x}>0 \text {. }
$$

Whenever any of these conditions are violated one of the eigenvalues has a positive real part and the steady state is unstable.

The first appearance of an instability always involves violating either condition (6) or (7). To show this it is useful to first note the Jacobian for the transformation from extensive to intensive variables. This is given by ${ }^{6}$

$$
J=\partial(s, x) / \partial(T, P)=(\alpha x)^{2}-C_{p} \kappa_{T} x / T,
$$

which is equivalent to

$$
J=\partial(s, x) / \partial(T, P)=-C_{V} \kappa_{T} x / T .
$$

Here $\kappa_{T}=(-1 / x)(\partial x / \partial P)_{T}$ is the isothermal compressibility, $\alpha=(1 / x)(\partial x / \partial T)_{P}$ is the thermal expansion coefficient, and $C_{P}$ and $C_{V}$ are heat capacities. Since we are considering a single phase system these quantities are always positive and finite.

Using this Jacobian we find that Eq. (5) becomes

$$
P_{x}=-\gamma / \kappa_{T} x<0,
$$

where $\gamma$ is the heat capacity ratio. This condition is always satisfied. Also, we now see that Eqs. (6) and (7) are sufficient to guarantee that Eq. (4) is satisfied. Thus, Eqs. (6) and (7) are both necessary and sufficient for stability. At the point where either of these conditions is first violated the system undergoes a bifurcation from stable to unstable behavior. However, these two bifurcations are quite different.

When condition (6) is first violated one eigenvalue is equal to zero. Beyond this point this root is positive and the system moves exponentially away from the unstable steady state. This conditions occurs only when there are multiple steady states, at least two of which are stable.

To show this we may use Eq. (8) to obtain the following equality:

$(\partial h / \partial T)_{P}=(J)[\partial(h, P) / \partial(s, x)]=\left(h_{x} P_{s}-h_{s} P_{x}\right) C_{V} \kappa_{T} x / T$

We therefore see that Eq. (6) is not satisfied when

$$
(\partial h / \partial T)_{P}>0 .
$$

Now consider a plot of $h$ vs $T$ for constant $P=P_{0}$. A steady state will occur at any temperature where $h=0$. If, at such a point, Eq. (9) is satisfied then the curve will have a positive slope at that point. However, we should require, on physical grounds, that heat flows in at sufficiently low temperatures and out at sufficiently high temperatures. This means that we have $h>0$ as $T \rightarrow 0$ and $h<0$ as $T \rightarrow \infty$. Thus, if there is an unstable steady state at which (9) is satisfied there will be two stable steady states at which it is not satisfied. A displacement from the unstable state will presumably evolve to one of the stable states.

It can be shown ${ }^{5}$ that when condition (7) is first violated (i.e., $P_{s} h_{x}=0$ ) there is a pair of pure imaginary eigenvalues. As $P_{s} h_{x}$ becomes positive these eigenvalues become complex conjugates with positive real parts. Thus, this instability corresponds to a growing oscillation.

To see the physical interpretation of this condition note that it is equivalent to saying that the steady state is unstable when

$$
h_{x} / P_{s}<0 .
$$

Using the Maxwell relation

$$
(\partial P / \partial s)_{x}=-(\partial T / \partial x)_{s},
$$

this becomes

$$
(\partial h / \partial T)_{s}>0 .
$$

Recall that this is to be evaluated at a steady state point, i.e., a point where $h=0$. When this instability condition is satisfied heat will flow into the system at high temperature and out at low temperature. This is, of course, what happens in a heat engine. It is necessary in order for a cyclic process to be capable of providing work. For our system, the increase in the concentration of the absorbing species with increased temperature makes it possible for this to occur.

To understand in more detail just how the heat flow drives the oscillations we consider the ratio of partial derivatives

$$
\begin{aligned}
& (\partial P / \partial x)_{h} /(\partial P / \partial x)_{s} \\
& =[\partial(P, h) / \partial(x, s)](\partial s / \partial h)_{x} /(\partial P / \partial x)_{s} \\
& =1-\left(P_{s} h_{x}\right) /\left(P_{x} h_{s}\right) .
\end{aligned}
$$

If all the stability conditions except (7) are satisfied this ratio will be greater than one. Consequently, as the volume increases the pressure will drop more rapidly along a curve for 
which $h=0$ than it will along an isentrope. This situation is shown in Fig. 3.

Imagine that the piston is moving outward past a point where $h=0$ and $P=P_{0}$. When the piston is moving rapidly the state of the fluid changes in a more nearly adiabatic (isentropic) manner than when the piston moves slowly. As the fluid expands the pressure drops and the piston decelerates. The path in the $P, x$ plane describing the state of the fluid drops toward the curve $h=0$ as heat flows out of the system. After the piston reverses its motion it accelerates inward until it passes the equilibrium point. Now, as the piston slows, heat flows in and the state of the fluid rises toward the curve $h=0$. The net result of this is that the pressure is higher on expansion than on compression so that work is done by the fluid on the piston. This causes the piston to move faster on each cycle. If the curve $h=0$ were to drop less steeply than the isentrope the opposite would be true and the oscillations would be damped.

\section{OPTIMAL CONTROL OF THE DISSIPATIVE ENGINE}

We turn now to the problem of finding the piston trajectory that produces the maximum average power and determining that power. We choose to maximize the average power because we envision an engine exposed to an essentially constant flux of energy, e.g., as light. In this case maximizing the power is equivalent to maximizing the fraction of the light energy converted into work.

The solution to this problem can be obtained by optimal control theory, ${ }^{7}$ which is a form of the calculus of variations. Solutions of problems of this type are usually in the form of a

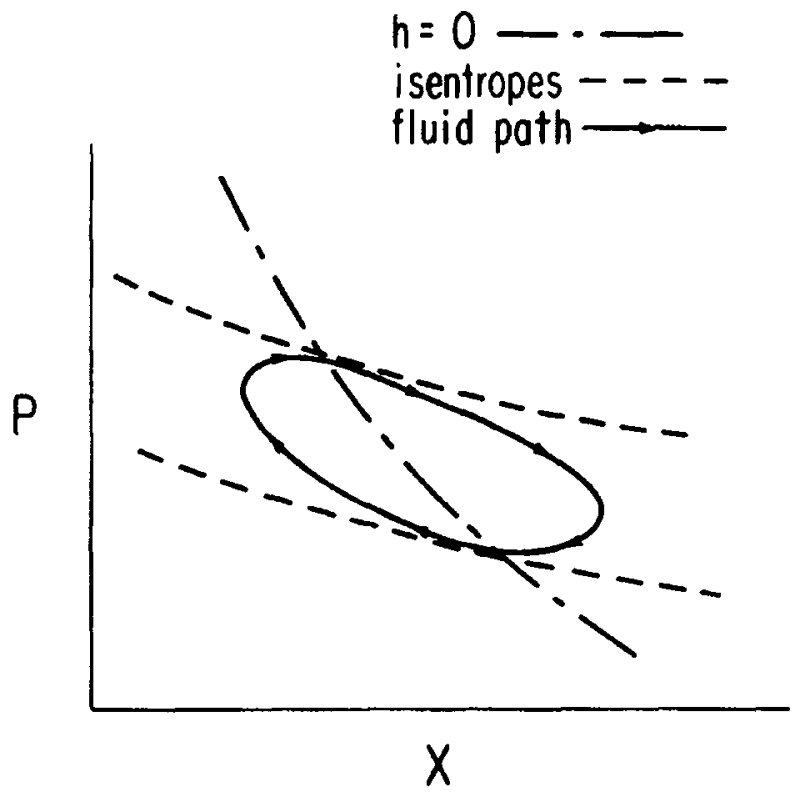

FIG. 3. Pressure-volume diagram for unstable oscillations. Due to heat flow the state of the fluid evolves away from the isentropes and toward the curve $h=0$. If the curve $h=0$ drops more steeply than the isentropes then work is done on the piston. system of differential equations which describe the optimal path as a function of time. However, in this particular problem, a constant of the motion determines the types of paths in thermodynamic state space (such as adiabats and isotherms) which may form branches of the optimal cycle. Piecing these branches together leads to a system of algebraic equations, the roots of which describe the optimal cycle.

An interesting feature of the way the problem is formulated here is that the only effect of the piston motion is to control the state of the fluid and, thereby the heat flows. The irreversibilities, available work, and net work done (in a cyclic process) depend only on the heat flows. Consequently it is not necessary to specify the manner in which work is extracted from the engine. This general approach should be useful in extending this treatment to systems, such as chemical oscillations, for which no mechanism for work extraction has yet been devised.

The problem is stated mathematically as follows. We seek the control function, $x(t)$, which maximizes the average power, $\Pi$, delivered to a work reservoir:

$$
\Pi=Q\left(t_{F}\right) / \tau\left(t_{F}\right),
$$

subject to the constraints

$$
\begin{aligned}
& d s / d t=h(s, x) / T(s, x), \\
& d Q / d t=h(s, x),
\end{aligned}
$$

and

$$
d \tau / d t=1,
$$

and the boundary conditions

$$
s(0)=s\left(t_{F}\right),
$$

and

$$
Q(0)=\tau(0)=0 .
$$

Here $t_{F}$ is the final time, $Q$ is the net heat flow into the engine, $h$ is the heat transfer function as in Sec. II, and $\tau$ is a dummy variable that is introduced so that the system is formally autonomous. As before $s$ and $x$ are the entropy and volume. In the appendix we transform the variables to ones which may be more useful in practical problems.

Equation (15) ensures cyclic operation of the engine. It is not necessary to specify the initial and final volumes since the control $x$ is allowed to change discontinuously. Consequently, the volume can be adjusted at the ends of the path without affecting the objective function.

This is a variational problem in Mayer form, i.e., the quantity to be extremized is expressed as a function of the final state of the system. This form is convenient for our problem since the objective function is a ratio of two functionals of the path. The final time $t_{F}$ is allowed to vary so that we may find its optimal value.

We begin by constructing the Hamiltonian ${ }^{7}$ from the right hand sides of the constraints, Eqs. (12)-(14), and a set of Lagrange multipliers $\lambda_{i}$ to get

$$
H=\lambda_{1} h / T+\lambda_{2} h+\lambda_{3} \text {. }
$$

It is a standard result of optimal control theory ${ }^{7}$ that a path which maximizes the objective function satisfies the following conditions: 
(1) The Lagrange multipliers obey Hamilton's equations

$d \lambda_{1} / d t=-\partial H / \partial s=-\left(\lambda_{1} / T+\lambda_{2}\right) h_{s}+\lambda_{1} h T_{s} / T^{2}$

$d \lambda_{2} / d t=-\partial H / \partial Q=0$,

$d \lambda_{3} / d t=-\partial H / \partial \tau=0$.

(2) Since the final time is not specified,

$$
H=0
$$

for the entire path.

(3) The Pontryagin Maximum Principle must be satisfied. This principle requires that

$0=\partial H / \partial x=\lambda_{1}\left(T h_{x}-h T_{x}\right) / T^{2}+\lambda_{2} h_{x}$.

(4) The "natural" boundary conditions are

$$
\begin{aligned}
& \lambda_{1}(0)=\lambda_{1}\left(t_{F}\right), \\
& \lambda_{2}=\partial \Pi / \partial Q, t=t_{F}, \\
& \lambda_{3}=\partial \Pi / \partial \tau, t=t_{F} .
\end{aligned}
$$

These last two conditions become [note Eqs. (19) and (20)]

$$
\lambda_{2}=1 / \tau_{F}
$$

and

$$
\lambda_{3}=-Q\left(t_{F}\right) /\left(\tau_{F}\right)^{2}=-\Pi \lambda_{2} .
$$

(5) As the fifth condition, $H, \partial H / \partial x$, and the $\lambda_{i}$ must be continuous even at "corners," i.e., points where $x$ is discontinuous.

These equations provide the necessary conditions for an extremal of the power. If more than one solution with $\Pi>0$ exists they must be examined to determine which is the absolute maximum.

We now determine the types of arcs that satisfy the conditions given above. Then we piece these arcs together to determine the full cycle and the maximum power.

Equations (17), (21), (22), and (24) provide us with three linear homogeneous equations in the three $\lambda_{i}$ 's; we wish to find the nontrivial solution. Rearranging (22) we obtain

$$
\lambda_{1} / \lambda_{2}=T^{2} /\left[h\left(T_{x} / h_{x}\right)-T\right] .
$$

Substituting (24) into (17), with $H=0$, and rearranging we get

$$
\lambda_{1} / \lambda_{2}=T(I-h) / h .
$$

Combining these and solving for $\Pi$ we obtain

$$
\Pi=h^{2} /\left[h-T\left(h_{x} / T_{x}\right)\right] \text {. }
$$

This provides us with a constant of the motion which is numerically equal to the average power.

Since this constant is a function of the thermodynamic state variables only, it defines a family of curves in the $s, x$ plane which may be parts of the optimal cycle. From (26) we see that if $h=0$ anywhere on one of these curves then $\Pi=0$ and $h=0$ everywhere on the curve. ${ }^{8}$ Since $h$ should remain finite this means that on a given arc of constant $I$ the sign of $h$ does not change. However, from Eq. (12) we see that $h$ must change sign if the entropy is to return to its original value. This means that the optimal cycle must contain at least two separate arcs, each with the same value of $\Pi$ but with different signs of $h$.
The only way for the system to move between two arcs with different signs of $h$ is for there to be a discontinuity in the control variable, $x$. This change in volume, instantaneous in the idealized world, can be given a physical interpretation as an arc which is completed in a time short compared to the time scales associated with the flow of heat into and out of the system. This is simply the definition of an adiabat. ${ }^{9}$ Since we assume that the fluid is in internal equilibrium (i.e., the engine is endoreversible $e^{4}$, these adiabats are also isentropes. Adiabats of this type have been noted previously in engine optimization problems. ${ }^{4,10}$

We have now determined that the optimal cycle may contain two types of arcs; constant $\Pi$ arcs [described by Eq. (26)] and (essentially) instantaneous adiabats. In order to piece together a complete cycle we must determine the points at which the adiabats occur and the constant $\Pi$ arcs they connect. These points must satisfy the continuity conditions on $H, \partial H / \partial x$, and $\lambda_{i}$. The first two quantities are continuous since they are both zero on arcs of constant $\Pi$ [Eqs. (17), (21), and (22)]. Obviously $\lambda_{2}$ and $\lambda_{3}$ are continuous by virtue of being constants [Eqs. (19) and (20)]. The continuity of $\lambda_{1}$ implies that the r.h.s. of Eq. (25) is continuous. Dividing Eq. (25) by (26) we obtain

$$
\lambda_{1} /\left(\lambda_{2} I\right)=g,
$$

where

$$
g=(T / h)^{2}\left(h_{x} / T_{x}\right)
$$

The continuity of $g$ and $I$ then serve to satisfy the corner conditions. In other words,

$$
I_{1}=I_{2}
$$

and

$$
g_{1}=g_{2},
$$

where the subscripts indicate the endpoints of the adiabats and $\Pi$ and $g$ are given by Eqs. (26) and (27).

An adiabat may be completely described by three quantities: for convenience, we take the entropy, which is constant along the adiabat, and the volumes at the two endpoints. We can therefore think of Eqs. (28) and (29) as defining a curve in the space of these three variables. Each point on this curve represents an adiabat and has some value of the power $\Pi$ associated with it. We are only interested in those adiabats for which $\Pi$ has its largest possible value. To find these adiabats we must maximize $\Pi$ with Eqs. (28) and (29) as constraints. "

In carrying out this process it is helpful to first obtain some additional expressions. Eliminating $\left(h_{x} / T_{x}\right)$ between Eqs. (26) and (27) we obtain

$$
g=T(1 / h-1 / I) \text {. }
$$

Taking derivatives with respect to $x$ and $s$ and making use of Eq. (27) we get

$$
g_{x}=T \Pi_{x} / \Pi^{2}
$$

and

$$
g_{s}=T \Pi_{s} / \Pi^{2}-f
$$

where

$$
f=T\left(T_{s} h_{x} / T_{x}-h_{s}\right) / h^{2} .
$$


Now we use Lagrange multipliers to adjoin the constraints of Egs. (28) and (29) to the function (either $\Pi_{1}$ or $\Pi_{2}$ ) that we are maximizing. This gives the augmented function

$$
F=\Pi_{1}+\lambda\left(\Pi_{1}-\Pi_{2}\right)+\mu\left(g_{1}-g_{2}\right) .
$$

Setting the variation of $F$ equal to zero and using Eqs. (31) and (32) we obtain

$$
\begin{aligned}
0=\delta f= & \left(\Pi_{1}\right)_{x}\left(1+\lambda+\mu T_{1} / \Pi_{1}^{2}\right) \delta T_{1} \\
& -\left(\Pi_{2}\right)_{x}\left(\lambda+\mu T_{2} / \Pi_{2}^{2}\right) \delta T_{2} \\
& +\left[\left(\Pi_{1}\right)_{s}\left(1+\lambda+\mu T_{1} / \Pi_{1}^{2}\right)\right. \\
& \left.-\left(\Pi_{2}\right)_{s}\left(\lambda+\mu T_{2} / \Pi_{2}^{2}\right)+\mu\left(f_{2}-f_{1}\right)\right] \delta s .
\end{aligned}
$$

We may choose $\lambda$ and $\mu$ so that the coefficients of $\delta T_{1}$ and $\delta T_{2}$ are zero. Then the coefficient of $\delta s$ must also be zero, i.e.,

$$
f_{1}=f_{2},
$$

where $f$ is given by Eq. (33). The roots of Eqs. (28), (29), and (34) define the adiabats which may occur in the optimal cycle.

These equations will not have a solution with positive power unless the condition for an oscillatory instability, Eq. (10), is satisfied. To see this we substitute Eq. (30) into (29) and rearrange to obtain

$$
h_{2}\left(T_{2}-T_{1}\right)=\Pi\left(T_{2}-T_{1} h_{2} / h_{1}\right)
$$

where $\Pi=\Pi_{1}=\Pi_{2}$. Now if $\Pi$ is positive and $h_{2}$ and $h_{1}$ have opposite signs (as they must in a cyclic process) we have

$$
h_{2}\left(T_{2}-T_{1}\right)>0 \text {. }
$$

This can only be satisfied if heat flows in $(h>0)$ at a higher temperature than it flows out. Therefore, as the system proceeds along an adiabat (isentrope) from $T_{1}$ to $T_{2}$ there must be an interval in which $(\partial h / \partial T)_{s}>0$.

The special case where $h$ is a function of $T$ alone serves to illuminate some features of the optimal cycle. In this case both $\Pi$ and $g$ are also functions of $T$ alone. Thus, the curves of constant $\Pi$ are isotherms and solving Eqs. (28) and (29) provides the temperatures, $T_{1}$ and $T_{2}$, of the allowable isotherms. In this case $f$ is identically zero [see Eq. (A3) in the appendix]. Consequently, Eq. (34) is satisfied for all adiabats connecting the two isotherms. The optimal cycle is shown in Fig. 4. It has the shape of a Carnot cycle but differs from the Carnot engine in that it is not reversible: the processes transferring heat between the engine and the external world are not reversible in this engine. In this regard, the engine described here is similar to the Curzon-Ahlborn engine. ${ }^{12}$

The average power is independent of the position of the adiabats. This is because the work done per cycle is equal to the area enclosed in the $s, T$ plane (Fig. 4). This in turn is proportional to the time spent on the isotherm since $h$, and therefore $d s / d t$, is constant on the isotherms. Therefore, the work per cycle is proportional to the period of the cycle and the power from the optimized engine is independent of the period.

This result may seen surprising in light of the results of Sec. II. We might expect that a resonance would occur at the frequency of the spontaneous oscillations. However the frequency of these oscillations is inversely proportional to the piston mass and therefore can be altered by changing the mass. Because of the nature of the assumptions about the

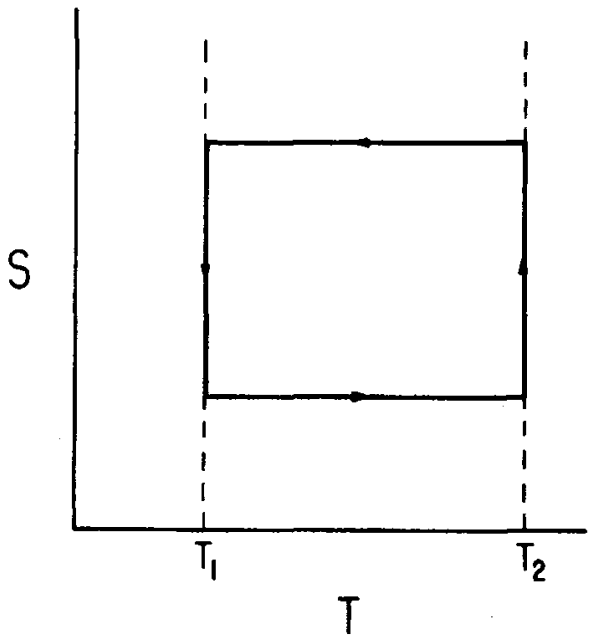

FIG. 4. The entropy-temperature diagram for the optimal cycle when the heat flow is a function of temperature alone. The arrows show the direction of the cycle which produces positive power.

control mechanism used in this section, the piston mass has no effect on the optimal motion. Consequently, there is no need for the optimal frequency to be the same as the natural frequency for some particular piston mass.

Among the cycles that produce the maximum power is one limiting case that consists of a single adiabat. This cycle takes place in zero time and produces zero work per cycle but has a power output equal to the maximum. If $h$ is allowed to depend on $x$ as well as $T$ we have no assurance that the corner conditions will be satisfied by more than one adiabat. When there is only one allowable adiabat the infinite frequency solution is the only solution. This solution provides an upper bound to the power output of the engine just as the Carnot cycle provides an upper bound to the efficiency of a conventional engine.

\section{DISSIPATIVE ENGINE WITH STEP FUNCTION ABSORPTION}

The analysis in this section is concerned with a heat transfer function which depends on temperature alone. In this case Eqs. (26) and (27) become

$$
\begin{aligned}
& I I=h^{2} /\left(h-T h_{T}\right), \\
& g=h_{r}(T / h)^{2} .
\end{aligned}
$$

In the type of heat engine discussed above the amount of light absorbed depends on the state of the working fluid. If this dependence is strong enough it can overcome the heat flow out of the engine and cause the net heat flow in to increase as the temperature increases. In this section we consider an idealized form of this behavior in which no light is absorbed below a certain temperature $T_{0}$ and all of the light is absorbed above that temperature. The heat flow out is assumed to be linear in the temperature. To simplify the equations we choose units in which the incident light energy flux and external temperature are both equal to one. This is equivalent to regarding the net heat flow $h$, the temperature $T$ and the thermal conductivity $k$ as being dimensionless ratios of these quantities to the incident flux and external 
temperature. We have for the heat flow function

$$
h=\theta\left(T-T_{0}\right)+k(1-T),
$$

where $\theta\left(T-T_{0}\right)$ indicates a step function. Since $h$ depends only on $T$ the optimal path will contain adiabatic jumps between isotherms at temperatures above and below $T_{0}$.

In an actual engine we might expect the conductivity to be different on the two isotherms. For example, it might depend on the area of the cylinder wall that is exposed to the working fiuid. Including such a volume dependence of $k$ would make the problem very complicated. However, we can get an idea of the effect it would have by allowing different conductivities on the two isotherms.

Letting $\beta$ indicate the ratio of the conductivities we have for the low $T$ isotherm

$$
h_{1}=\beta k\left(1-T_{1}\right)
$$

and for the isotherm at high $T$

$$
h_{2}=1+k\left(1-T_{2}\right) \text {. }
$$

Using Eqs. (35) and (36) we get the corner conditions

$$
\begin{aligned}
& \Pi_{1}=\left(h_{1}\right)^{2} /(\beta k)=\left(h_{2}\right)^{2} /(k+1)=\Pi_{2}, \\
& g_{1}=-\beta k\left(T_{1} / h_{1}\right)^{2}=-k\left(T_{2} / h_{2}\right)^{2}=g_{2} .
\end{aligned}
$$

Eliminating $h_{2} / h_{1}$ we find that

$$
(k+1) / \beta k=\left(h_{2} / h_{1}\right)^{2}=\left(T_{2} / T_{1}\right)^{2} / \beta,
$$

which reduces to

$$
T_{2} / T_{1}=r=(1+1 / k)^{1 / 2} \text {. }
$$

Thus, the temperature ratio depends only on the high temperature conductivity. Since $T_{1}>1$ this also provides a lower bound to the high temperature achieved in the cycle.

To find the isotherm temperatures we substitute Eqs. (37) and (38) into (39) to get

$$
\left(T_{1}-1\right)(\beta k)^{1 / 2}=\left[k\left(1-T_{2}\right)+1\right] /(k+1)^{1 / 2},
$$

which, after using Eq. (41), becomes

$$
\left(T_{1}-1\right) \sqrt{\beta}=r-T_{1} \text {. }
$$

From this we find

$$
\begin{aligned}
& T_{1}=(r+\sqrt{\beta}) /(1+\sqrt{\beta}), \\
& T_{2}=r(r+\sqrt{\beta}) /(1+\sqrt{\beta}) .
\end{aligned}
$$

From Eqs. (37) and (39) we obtain the average power

$$
\Pi=\Pi_{1}=\beta k\left(1-T_{1}\right)^{2} \text {. }
$$

Using Eq. (42) we transform this to

$$
\Pi=\beta k[(r-1) /(1+\sqrt{\beta})]^{2} .
$$

From Eq. (41) we have

$$
k=1 /(r-1)(r+1),
$$

so we get for the power,

$$
\Pi=\left[\beta /(1+\sqrt{\beta})^{2}\right](r-1) /(r+1) .
$$

From this we see that the power is maximized by large values of $\beta$ and $r$ (small $k$ ). Also, from Eqs. (42) and (43) we see that increasing $\beta$ with $r$ fixed decreases $T_{1}$ and $T_{2}$. In fact, as $\beta \rightarrow \infty, T_{1} \rightarrow 1$ and $T_{2} \rightarrow r$. Large values of $r$ produce large values of both high and low temperatures.

In a practical engine the maximum temperature is like- ly to be limited. From the above remarks we see that the largest power, for a given value of $T_{2}$, will occur as $\beta \rightarrow \infty$. From Eqs. (44) and (43) we obtain for this limit

$$
\Pi=(r-1) /(r+1)=\left(T_{2}-1\right) /\left(T_{2}+1\right) .
$$

Thus, the fraction of incident light converted to work is smaller than the Carnot efficiency. This is due to heat leakage from the engine on the high $T$ isotherm.

Another case of interest is $\beta=1$ so that the conductivity is the same on both isotherms. In this case

$$
\Pi=(1 / 4)(r-1) /(r+1),
$$

and, from Eq. (43),

$$
r=(1 / 2)\left[-1+\left(1+8 T_{2}\right)^{1 / 2}\right] \text {. }
$$

Since $r>1$ [Eq. (41)] $\Pi$ is always less than 0.25 . This is due to both heat leak on the high $T$ isotherm and finite time spent at low $T$ where no light is absorbed. Plots of $\Pi$ vs $T_{2}$ for these two cases are shown in Fig. 5.

\section{DISSIPATIVE ENGINE WITH CHEMICAL REACTION}

In this section we replace the step function of the previous section with a function describing absorption due to a chemical reaction. To keep $h$ a function of $T$ alone we consider a pure isomerization reaction and a constant thermal conductivity, $k$. The isomerization reaction might be a ring opening reaction of the type ${ }^{13}$

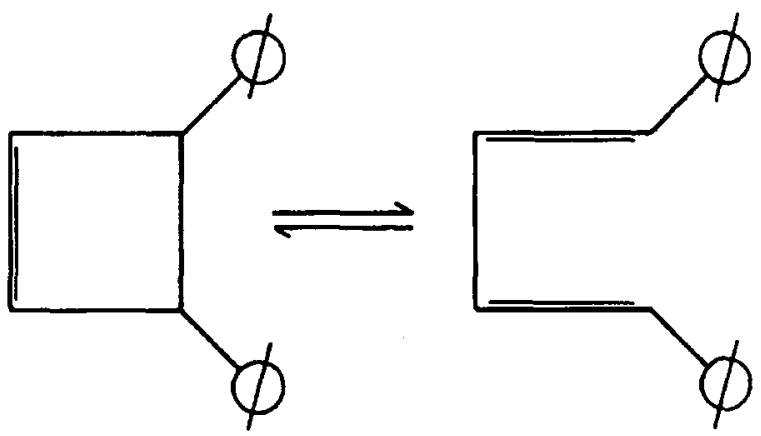

where the species on the right is the absorbing, high temperature species.

For this engine the heat flow is given by

$$
h=[(1-\exp (-b f)]+k(1-T),
$$

where the fraction of reacting material in the absorbing form is given by

$$
f=1 /[1+\exp (a+\epsilon / T)] .
$$

Here $b$ is the dimensionless product of the density, path length, and absorption coefficient, $a$ is the entropy change in the reaction, and $\epsilon$ is the heat of reaction. These are in units in which the gas constant, ambient temperature and incident energy flux all equal one.

As in the previous section $k$ determines the upper bound on the cycle temperatures; from Eq. (47) we see that all temperatures for which $h=0$ must lie in the range $1<T<1+1 / k . b$ determines the maximum fraction of light absorbed; for efficient operation we should have $b>1$. $\epsilon$ determines how sharp the transition between the absorbing and nonabsorbing forms is and $a$ determines the temperature 


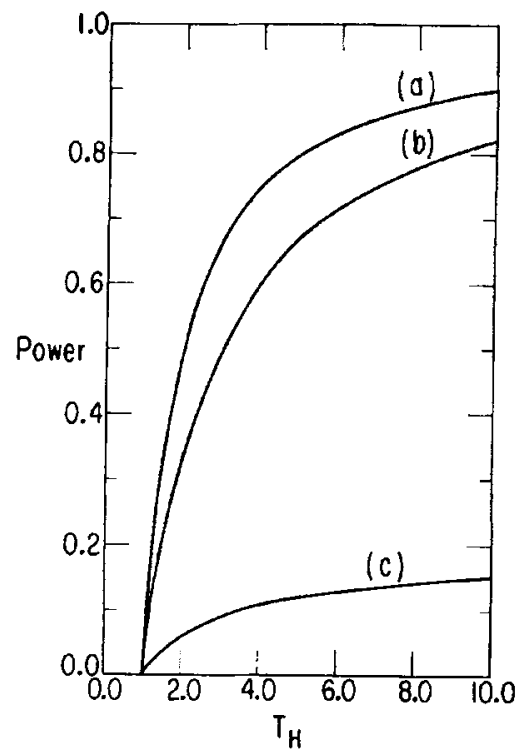

FIG. 5. Power as a function of the higher isotherm temperature for three cases: (a) reversible (Carnot) engine, (b) dissipative engine with $\beta \rightarrow \infty$ [Eq. (19)], (c) the case $\beta=1$ [Eq. (20)]. The incident energy flux and external temperature are set to one.

$T_{0}$ at which this transition takes place. For the purpose of this discussion we define $T_{0}$ as the temperature at which one half of the light is absorbed.

The heat flow function given in Eq. (47) will satisfy the instability conditions, Eq. (10), as long as there are multiple roots to $h=0$. The middle root corresponds to the steady state at which $h_{T}>0$. Figure 6 shows a sketch of the two terms comprising $h$ for different values of $a$. We see that if the maximum slope is large enough (i.e., $\epsilon$ is large enough) there should be a range of values of $a$ for which $h=0$ has three roots. We assume that $h$ has only one inflection point so that there are at most three roots.

The analysis of this model requires two stages. First, for a given set of parameters, we solve the corner conditions to find the maximum power. This requires finding the intersections of the curves $Y=0$ and $Z=0$, where

$$
Y\left(T_{H}, T_{L}\right)=\Pi\left(T_{H}\right)-I\left(T_{L}\right)
$$

and

$$
Z\left(T_{H}, T_{L}\right)=g\left(T_{H}\right)-g\left(T_{L}\right),
$$

with $T_{L}$ and $T_{H}$ the low and high temperatures of the cycle. We need a numerical procedure that will find all of these

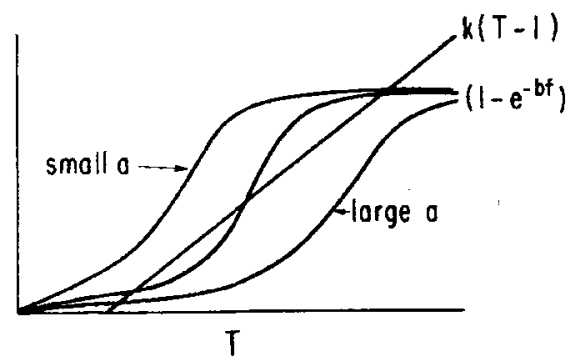

FIG. 6. The intersections of the curves $k(T-1)$ and $1-\exp (-b f)$ for the increasing values of $a$. intersections. Then we must examine the effect of the parameters on the power.

Examination of Eqs. (35) and (36) indicates that $h_{T}<0$ on both isotherms. The high temperature isotherm must therefore lie between a temperature $T_{3}$ at which $h$ is a maximum and the highest temperature root of $h=0$, which we designate as $T_{4}$. Similarly, $T_{L}$ must lie between $T_{1}$ (the lowest $T$ at which $h=0$ ) and $T_{2}$, at which $h-T h_{T}=0$. Using these conditions on $T_{1}$ and $T_{2}$ we find that, at a point of intersection, the ratio of the slopes of the curves $Y=0$ and $Z=0$ is

$$
\left(\partial T_{H} / \partial T_{L}\right)_{Y} /\left(\partial T_{H} / \partial T_{L}\right)_{z}=T_{H} / T_{L}>1 .
$$

Consequently, at any intersection the curve $Y=0$ is descending faster than the curve $Z=0$. Thus, there is at most one intersection.

This intersection can be located by the following iterative procedure:

(1) Let $T_{H}=\left(T_{3}+T_{4}\right) / 2$.

(2) Find $T_{L}$ to satisfy $Y=0$.

(3) Evaluate $Z\left(T_{H}, T_{L}\right)$

(4) If $Z>0$ let $T_{4}=T_{H}$. If $Z<0$ let $T_{3}=T_{H}$.

(5) Return to step (1) and repeat until the interval $T_{3}, T_{4}$ is sufficiently small.

The results of these calculations for various sets of parameters are tabulated in Table $I$ and plotted in Fig. 7. All of these results shown there are for $b=10$. As long as $b$ is greater than 5 it has little effect on the maximum power.

As can be seen from the figure, increasing $\epsilon$ from 10

\begin{tabular}{|c|c|c|c|c|c|c|}
\hline \multicolumn{7}{|c|}{ Step function heating: $\Pi=7.58, T_{H}=2.69$} \\
\hline \multirow{2}{*}{$\begin{array}{l}k=0.40 \\
T_{0}\end{array}$} & \multicolumn{2}{|c|}{$\epsilon=10$} & \multicolumn{2}{|c|}{$\epsilon=17$} & \multicolumn{2}{|c|}{$\epsilon=25$} \\
\hline & $\bar{\Pi}$ & $\overline{\dot{T}_{H}}$ & $\Pi$ & $\overline{T_{H}}$ & $\Pi$ & $\overline{T_{H}}$ \\
\hline 1.750 & 3.02 & 2.98 & 6.31 & 2.76 & 7.15 & 2.71 \\
\hline 1.875 & 4.20 & 2.87 & 6.80 & 2.74 & 7.40 & 2.70 \\
\hline 2.000 & 4.45 & 2.82 & 6.91 & 2.75 & 7.47 & 2.70 \\
\hline 2.125 & 3.98 & 2.80 & 6.75 & 2.80 & 7.42 & 2.73 \\
\hline 2.250 & 2.47 & 2.78 & 6.03 & 2.87 & 7.17 & 2.79 \\
\hline \multicolumn{7}{|c|}{ Step function heating: $I=10.51, T_{H}=4.23$} \\
\hline$k=0.20$ & \multicolumn{2}{|c|}{$\epsilon=10$} & \multicolumn{2}{|c|}{$\epsilon=17$} & \multicolumn{2}{|c|}{$\epsilon=25$} \\
\hline$T_{0}$ & $\bar{\Pi}$ & $\overline{T_{H}}$ & $\bar{\Pi}$ & $\overline{T_{H}}$ & $\bar{n}$ & $\overline{T_{11}}$ \\
\hline 2.00 & 3.40 & 4.98 & 8.18 & 4.43 & $\ldots$ & $\ldots$ \\
\hline 2.25 & 5.64 & 4.68 & 9.14 & 4.35 & 10.00 & 4.27 \\
\hline 2.50 & 6.66 & 4.52 & 9.67 & 4.31 & 10.28 & 4.24 \\
\hline 2.625 & 5.79 & 4.46 & 9.74 & 4.31 & 10.36 & 4.24 \\
\hline 2.75 & 6.72 & 4.41 & 9.76 & 4.33 & 10.39 & 4.24 \\
\hline 3.00 & 5.87 & 4.30 & 9.34 & 4.42 & 10.30 & 4.30 \\
\hline 3.25 & 4.06 & 4.19 & $\cdots$ & $\cdots$ & 9.92 & 4.43 \\
\hline \multicolumn{7}{|c|}{ Step function heating: $I=13.42, T_{H}=7.16$} \\
\hline$k=0.10$ & \multicolumn{2}{|c|}{$\epsilon=10$} & \multicolumn{2}{|c|}{$\epsilon=17$} & \multicolumn{2}{|c|}{$\epsilon=25$} \\
\hline$T_{0}$ & $\bar{\Pi}$ & $\overline{T_{H}}$ & $\bar{n}$ & $\overline{T_{H}}$ & $\bar{n}$ & $\overline{T_{H}}$ \\
\hline 2.50 & 5.07 & 8.62 & 10.52 & 7.60 & 12.07 & 7.36 \\
\hline 3.00 & 7.51 & 8.01 & 11.69 & 7.41 & 12.77 & 7.25 \\
\hline 3.50 & 8.32 & 7.61 & 12.22 & 7.35 & 13.13 & 7.20 \\
\hline 4.00 & 8.03 & 7.20 & 12.12 & 7.38 & 13.22 & 7.22 \\
\hline 4.50 & 6.88 & 6.77 & 11.24 & 7.44 & 12.92 & 7.36 \\
\hline 5.00 & 5.12 & 6.34 & 9.37 & 7.49 & 11.99 & 7.61 \\
\hline
\end{tabular}
(about $6 \mathrm{kcal} / \mathrm{mole}$ for an ambient temperature of $300 \mathrm{~K}$ ) to

TABLE I. Data for engine with chemical reaction. 


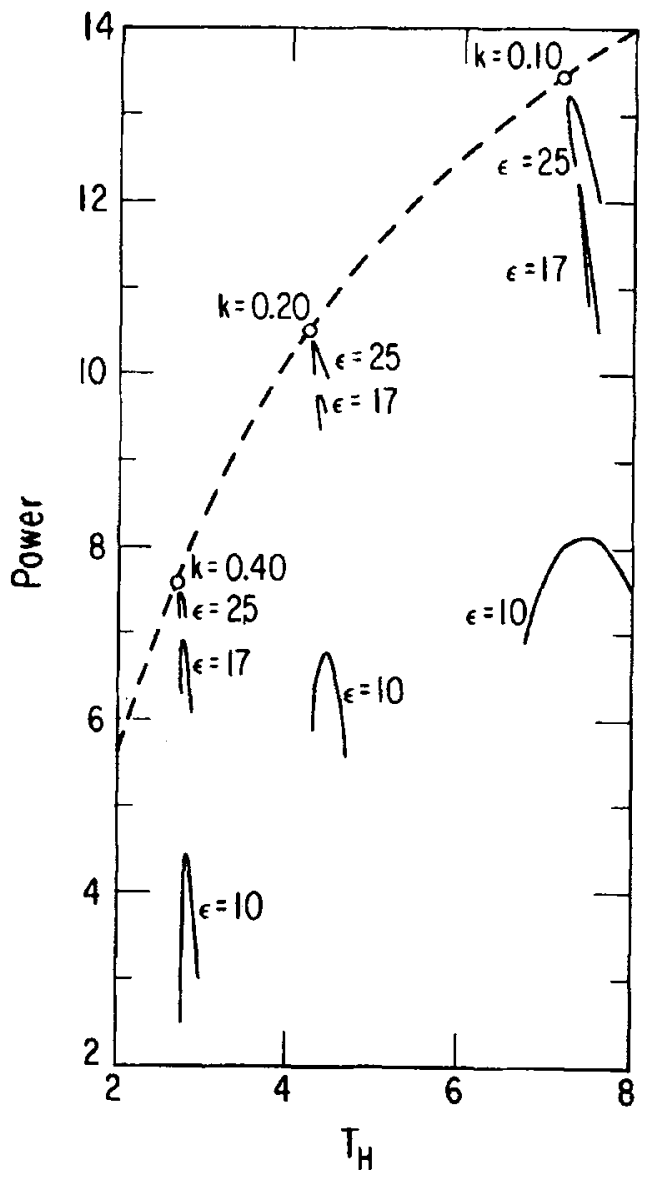

FIG. 7. Power for the dissipative engine with chemical reaction plotted vs $T_{H}$ as $T_{0}$ is varied. The broken curve is for the step function heating. Data are given in Table $\mathrm{I}$.

25 (about $15 \mathrm{kcal} / \mathrm{mole}$ ) increases the power from about $60 \%$ to about $99 \%$ of that achieved with the step function. Larger values of $\epsilon$ have little effect. The value of $\epsilon$ has little effect on the high temperature.

Decreasing $k$ has the effect of increasing both the average power and the high temperature of the cycle. This is in agreement with the effect that $k$ has with the step function heat input. With the chemical reaction the optimal maximum temperature is slightly higher than with the step function and the minimum temperature is slightly lower.

The value of $T_{0}$ is quite important. If it is too small there will be either significant absorption at the low temperature or too small a heat flow out. In the latter case a disproportionate amount of time will be spent at low $T$. If $T_{0}$ is too high there will be either unabsorbed light or too large a heat flow out at high $T$. The best value of $T_{0}$ turns out to be roughly the geometric mean of the high and low temperatures.

Instead of a pure isomerization reaction we might wish to use a reaction that involves a change in the mole number. An example would be the dimerization reaction

$$
2 \mathrm{NO}_{2} \leftrightarrow \mathrm{N}_{2} \mathrm{O}_{4} \text {, }
$$

where $\mathrm{NO}_{2}$ absorbs and is favored at high temperatures. For such a reaction the equilibrium constant (in mole fractions) depends on the volume as well as the temperature. However, the temperature dependence should dominate since it is exponential. Thus, we might expect this system to behave similarly to the isomerization reaction but with the value of $T_{0}$ depending on the volume. $T_{0}$ could be adjusted to a favorable value by changing the density of $\mathrm{NO}_{2}$. From the results of the previous section we should expect that introducing a volume dependence in the heat flow out should enable us to achieve much better efficiencies.

The trajectory needed to produce the maximum power may be quite difficult to produce. We now consider the same sort of engine as discussed previously but with the piston required to move sinusoidally. Then the volume will be specified as a function of time. To find the temperature trajectory and the power we need to use a specific equation of state.

If the working fluid consisted entirely of the reacting materials the compression ratio required would be prohibitively large. This is primarily because the compression would have to provide the heat of reaction. Also, if the absorbing species has a large number of internal degrees of freedom the heat capacity ratio will be near one. These problems can be circumvented by assuming that the absorbing species is diluted with a large amount of a buffer gas. The buffer gas alone will then determine the equation of state.

If the buffer is an ideal gas the equations of motion for this system are

$$
\begin{aligned}
& d W / d t=T v / x, \\
& d T / d t=[h(T)-T v / x] / C_{v}, \\
& v=(\omega / 2)\left(1-x_{0}\right) \sin (\omega t), \\
& x=x_{0}+\left(1-x_{0}\right)[1-\cos (\omega t)] / 2,
\end{aligned}
$$

where $W$ is the work done by the engine, $h(T)$ is given by Eq. (47), $\omega$ is the frequency, $x_{0}$ is the maximum volume (compression ratio), $C_{v}$ is the constant volume heat capacity, and the gas constant and minimum volume are set equal to one.

These equations were integrated using a Runge-Kutta routine. For a given period the compression ratio was varied until the maximum power was obtained. This was repeated for various values of the period and with heat capacities of $3 R / 2$ and $5 R / 2$. For the results presented here the parameters used in the heat transfer function, Eq. (47), were $\epsilon=25$, $b=10, k=0.2$, and $T_{0}=2.75$.

The results of the calculations are presented in Table II. For comparison, the power and maximum temperature are given for a step heating function with the same value of $k$ and for the optimal solution with the same values of the parameters describing the chemical reaction.

From the table we see that the sinusoidal motion requires a somewhat higher maximum temperature than the optimal motion. With $C_{v}=3 R / 2$ about $68 \%$ of the maximum power is produced, with $C_{v}=5 R / 2$ this drops to $60 \%$.

Varying the period over a range of more than six orders of magnitude does not change the power output. No calculations were done with periods of less than $10^{-5}$. With periods of 10 or above the optimal compression ratio begins to increase and the maximum power begins to drop off slightly.

The independence of the power output from the period 
TABLE II. Results with sinusoidal pisțon motion.

\begin{tabular}{llll}
\hline \hline Period & $\begin{array}{l}\text { Compression } \\
\text { ratio }\end{array}$ & $T_{H}$ & Power \\
\hline Step heating function & 4.23 & 10.51 \\
Optimum & & 4.24 & 10.39 \\
$10^{-5}$ & 4.20 & 4.57 & 7.04 \\
$10^{-3}$ & 4.20 & 4.57 & 7.04 \\
0.1 & 4.20 & 4.57 & 7.04 \\
1.0 & 4.20 & 4.57 & 7.04 \\
10.0 & 4.50 & 4.51 & 7.03 \\
20.0 & 5.75 & 4.60 & 7.00 \\
30.0 & 7.75 & 4.60 & 6.92 \\
1.0 & 3.00 & 3.70 & 6.04 \\
1.0 & 4.00 & 4.39 & 7.00 \\
1.0 & 4.20 & 4.57 & 7.04 \\
1.0 & 4.50 & 4.84 & 6.98 \\
1.0 & 5.00 & 5.33 & 6.62 \\
a & 8.50 & 4.53 & 6.21 \\
\hline \hline
\end{tabular}

- Period ranging from $10^{-4}$ to 1.0 , with $C_{v} / R=2.5$. For all other entries $C_{w} / R=1.5$.

is identical to the situation with the optimal trajectory. This feature would be very desirable in a practical engine since it would eliminate the need for a transmission in matching the engine torque to the load. A working engine would presumably be designed to operate near the lowest frequency which does not require an increased compression ratio. This would minimize losses not considered here, such as those due to friction and finite rates of reaction.

\section{SUMMARY}

A dissipative engine of the sort considered here cannot be operated reversibly. Consequently, its theoretical efficiency will always be less than the reversible efficiency of a conventional engine. However, for this very reason, we expect that a real dissipative engine would come closer to achieving its theoretical efficiency than a conventional engine would. This is because as soon as we begin to operate the conventional engine at a finite rate we introduce losses due to heat transfer effects. These losses are already included in the idealized model of the dissipative engine.

The dissipative engine would be mechanically simple. It requires no mechanisms, such as valves or shutters, to connect it to its reservoirs. This job is carried out by the chemical reaction in the working fluid. This mechanical simplicity should be especially valuable for a solar engine since, in that case, the fuel is free. As a result, the dominant costs will be those due to building and maintaining the engine.

There are a number of questions that must be answered if we are to build an engine that operates on this principle. First of all we would need to choose the working fluid. Then we would have to design the mechanical features of the engine such as the dimensions of the cylinder, amount of insulation, and details of the piston motion. A traditional engineering approach to designing such an engine would have to rely initially on intuition to make these choices. With experience the choices would be improved but changes would still have to be made on a largely trial and error basis. A great deal of effort would be required before we would have a reasonably clear idea of what the prospects for success are.

The results presented here provide a means of streamlining this process. We can carry out these analyses without knowing all the details of the engine. All that is required is a knowledge of the heat transfer function and the equation of state of the fluid. Given that information we can determine if there is a range of temperatures for which, at a steady state, the net heat flow inward increases with increasing temperature. Any candidate system for which this condition is not satisfied at reasonable temperatures can be eliminated.

Next the results of Sec. III can be used to determine the maximum power. This requires the solution of a set of algebraic equations which also provide the extreme temperatures of the cycle. These results can be used to choose the most promising candidates for future development. As that development is carried out, the maximum power will provide a standard for comparison.

From the results of Sec. IV and V we see that, with a heat transfer function that depends on temperature alone, the optimal cycle is a Carnot cycle with an arbitrary period. We have a limiting efficiency of $25 \%$ and with reasonable operating temperatures an efficiency of about $10 \%$. These figures are comparable to the limiting and actual efficiencies of solar cells. An actual engine with such a heat transfer function would not do this well. For example, sinusoidal piston motion reduces the efficiency to about two-thirds of the optimum. Other losses, such as friction and reflective losses, would cause further reductions in efficiency. On the other hand, introducing a volume dependence in the heat transfer has the potential to produce large improvements in the efficiency.

Finally, we note a possible application of these results to chemically sustained oscillations. The basic reason that any heat engine operates periodically is so that the temperature of the working fluid is higher when heat flows in than it is when heat flows out. This reduces the potential difference between the high $T$ source and the engine and between the engine and the low $T$ sink. This reduction in the irreversible losses becomes part of the potential difference available to produce work.

A similar situation might be expected to occur in a chemical system. The heat reservoirs could be replaced by large reservoirs of reactants and products. The overall reaction could proceed by means of intermediate species which could also react with a different system to drive a reaction against a chemical potential gradient. It may well be advantageous for the concentrations of the intermediates to oscillate, thus periodically reducing the chemical potential differences between the intermediates and the reactants and products reservoirs. We might speculate that biochemical oscillations occur for reasons of this sort.

After this manuscript had been submitted, we learned from Dr. John Wheatley of another proposal ${ }^{14}$ for an engine that "depends intrinsically for its operation on irreversible processes" and is therefore an engine based on a dissipative process. Wheatley $e t$ al. have constructed an engine of this type, based on a thermoacoustic effect in a gas $\left({ }^{4} \mathrm{He}\right)$ contained in a chamber with parallel plates, subjected to a ther- 
mal gradient. While their work and ours take altogether different approaches to the analysis of the problem, and the physico-chemical model used to illustrate our approach looks altogether different from a thermoacoustic engine, the two lines of work have clearly converged onto the common idea of extracting work from a system capable of spontaneous oscillations.

\section{ACKNOWLEDGMENTS}

We would like to thank Dr. John Wheatley for communicating the results of his work to us prior to its publication. This research was carried out with the support of a grant from the Division of Basic Energy Sciences of the United States Department of Energy.

\section{APPENDIX: CHANGE OF VARIABLES}

Throughout this paper the heat flow and temperature are assumed to be known as functions of $s$ and $x$. In practice, we are more likely to have $h$ given as a function of $T$ and $x$. Also, the equation of state is more likely to be known in the form of the standard partial derivatives: the heat capacity $\left(C_{v}\right)$, compressibility $\left(\kappa_{\mathrm{T}}\right)$, and thermal expansion coefficient $(\alpha)$. Here we convert the expressions for $I$ [Eq. (26)], $g$ [Eq. (27)] and $f$ [Eq. (33)] into this form.

First we must note some partial derivative relations ${ }^{6}$ :

$$
\begin{aligned}
& T_{s}=(\partial T / \partial s)_{x}=T / C_{v}, \\
& h_{s}=(\partial h / \partial s)_{x}=\left(T / C_{v}\right)(\partial h / \partial T)_{x}, \\
& \alpha / \kappa_{T}=(\partial P / \partial T)_{x}=(\partial s / \partial x)_{T}, \\
& T_{x}=(\partial T / \partial x)_{s}=-(\partial T / \partial s)_{x}(\partial s / \partial x)_{T}=-T \alpha / C_{v} \kappa_{T}, \\
& h_{x} / T_{x}=(\partial h / \partial T)_{s}=(\partial h / \partial T)_{x}+(\partial h / \partial x)_{T}(\partial x / \partial T)_{s}, \\
& h_{x} / T_{x}=(\partial h / \partial T)_{x}-\left(C_{v} \kappa_{T} / T \alpha\right)(\partial h / \partial x)_{T} .
\end{aligned}
$$

Using these expressions we obtain

$$
\begin{aligned}
& \Pi=h^{2} /\left[h+\left(C_{v} \kappa_{T} / \alpha\right)(\partial h / \partial x)_{T}-T(\partial h / \partial T)_{x}\right] \\
& g=T\left[T(\partial h / \partial T)_{x}-\left(C_{v} \kappa_{T} / \alpha\right)(\partial h / \partial x)_{T}\right] / h^{2} \\
& f=-\left(T \kappa_{T} / h^{2} \alpha\right)(\partial h / \partial x)_{T} .
\end{aligned}
$$

Since the entropy does not appear explicitly in these expressions we must add to the conditions (28), (29), and (34) the condition

$$
s\left(x_{1}, T_{1}\right)=s\left(x_{2}, T_{2}\right)
$$

\footnotetext{
'A. Nitzan and J. Ross, J. Chem. Phys. 59, 241 (1973).

${ }^{2}$ V. Fairén and J. Ross, J. Chem. Phys. 75, 5485 (1981).

${ }^{3}$ M. Mozurkewich and R. S. Berry, Proc. Nat. Acad. Sci. USA 78, 1886

(1981); M. Mozurkewich and R. S. Berry, J. Appl. Phys, 53, 34 (1982).

${ }^{4} M$. Rubin, Phys. Rev. A 19, 1272, 1277 (1979); 22, 1741 (1980).

'B. Porter, Stability Criteria for Linear Dynamical Systems (Oliver and Boyd, Edinburgh, 1967).

${ }^{6}$ H. B. Callen, Thermodynamics (Wiley, New York, 1960), pp. 128-130.

'A. E. Bryson and Y. C. Ho, Applied Optimal Control (Wiley, New York, 1975): A. P. Sage, Optimum Systems Control (Prentice Hall, Englewood Cliffs, New Jersey, 1968).

If $\Pi$ remains nonzero when $h=0$ then $\lambda_{1} / \lambda_{2}$ must diverge. This implies that $\lambda_{2}=0$ [Eqs. (18) and (23)] which, with Eqs. (24), (17), and (21), implies that $\lambda_{1} h=0$ everywhere on the curve.

${ }^{9}$ L. C. Woods, Thermodynamics of Fluid Systems (Clarendon, Oxford, 1975), p. 6.

${ }^{10}$ P. Salamon, A. Nitzan, B. Andresen, and R. S. Berry, Phys. Rev. A 21, 2115 (1980).

"The usual procedure in optimal control theory is to use the boundary conditions to determined an additional condition on the adiabats. This procedure gives the same results as obtained here.

${ }^{12}$ F. L. Curzon and B. Ahlborn, Am. J. Phys. 43, 22 (1975).

${ }^{13}$ We thank N. C. Yang for suggesting this reaction.

14J. Wheatley, T. Hofler, G. W. Swift, and A. Migliori, Phys. Rev. Lett. (to be published).
} 